\title{
The Time Variation of the Maize Yield in Precise Operation Section
}

\author{
Yueling Zhao ${ }^{1, a^{*}}$, Haiyan Han ${ }^{2, ~ c}$, Liying Cao ${ }^{1}$ and Guifen Chen ${ }^{1, d}$ \\ ${ }^{1}$ Jilin Agricultural University, Changchun, 130118, China; \\ ${ }^{2}$ Changchun University, Changchun, 130000, China \\ azyueling@163.com, ${ }^{\text {b } 275714495 @ \text { qq.com, }{ }^{c} 453876175 @ q q . c o m,{ }^{d} 1045093555 @ q q . c o m}$
}

\begin{abstract}
Keywords: Time variation; Maize yield; Precise operation section; Prediction model
\end{abstract}
\begin{abstract}
In this paper, a variety of curve estimation method is used to predict the time series characteristics of Maize Yield in more than 20 years, and it is found that each prediction method has its applicable conditions and applicable scope. Comparison of the parameters of the estimated parameters of the four kinds of curves found that the effect of the three curve estimation is optimal, so the function of the three Cubic is more appropriate to observe the value of the fitting. If the ARIMA model is used to predict the yield of maize, the AIC $(2,1,1)$ (ARIMA) has achieved good results in the study. The conclusions can provide some guidance and practical significance for the current and even future production management decision.
\end{abstract}

\section{Introduction}

With precise technique continuously expanded and applied in agricultural production in our country, so that crop yields continually improved, under the food problem more and more attention of figure, how to forecast the future study of grain output over a period of time also gradually become a research hotspot. To understand the relationship between maize yield and time series in the precise areas, and the result is quite necessary to make a comprehensive analysis and application, it is one of the effective utilization of agricultural resources and technology. The paper analysis and forecasting modes through the application of a variety of algorithms for time series analysis on maize yield.On the one hand can be scientific, reasonable and effective evaluation on it, and the agricultural production workers can not only improve the utilization efficiency of soil nutrients, and can save the resources, obtain better economic benefits, to protect the limited agricultural ecological resources and the quality.

\section{Materials and Methods}

The Mode of Data Acquisition. This study is based on the data collected from the Jilin statistical yearbooks, we can collecte some information from the data, the time is from 1980 to 2011. the original data of the data analysis. In the actual data analysis, using this data as the original data to construct the time series prediction model. The results are as follows Table1.

Curve Estimation Method. The relationship between the properties of crop yield is interrelated and mutual restraint, and the characteristics of the attributes are not isolated and isolated in nature, there may be some correlation between them ${ }^{[1]}$. Correlation is measured by the degree of correlation between two or more variables. Correlation analysis can be carried out only with a correlation index. The study of the correlation between soil nutrients can reveal the relationship between the soil properties and the coordination effect between the indicators. Regression analysis is based on the change of the number of independent variables to predict the dependent variable, it is the regression equation as a prediction model ${ }^{[2]}$. This chapter mainly focused on the correlation between the yield and time series of soil properties in the precision work area, and established the model of the relationship between crop yield and time. The curve estimation methods commonly used flinear regression, nonlinear regression and trend extrapolation. in the analysis o

Auto Regressive Moving Average Model (ARIMA). Because of all kinds of things that exist in nature, are more or less interference and influence by external factors, so time series representation 
of things in real life are rarely completely smooth, they may show some "seasonal", that is to say in a certain cycle is changing, in order to solve the problem of unbalanced prediction sequence of the famous statistician Boakes and Jenkins put forward the sum of autoregressive moving average model (ARIMA model) is also called Box-Jenkins model or Boakes Jenkins Fa, also known as differential autoregressive moving average model ${ }^{[3-6]}$. The essence of this model is the application of the differential technology, the original time series smoothing process, namely the ARIMA model is a combination of differential operation and model ARMA ${ }^{[4-7]}$.

Table 1 Statistical table of maize yield from 1980 to 2011

\begin{tabular}{llllllllllll}
\hline code & year & yield & code & year & yield & code & year & yield & code & year & yield \\
& & & & & & & & & & & \\
\hline 1 & 1980 & 859.60 & 9 & 1988 & 1693.25 & 17 & 1996 & 2326.60 & 25 & 2004 & 2510.00 \\
2 & 1981 & 921.91 & 10 & 1989 & 1351.29 & 18 & 1997 & 1808.30 & 26 & 2005 & 2581.21 \\
3 & 1982 & 1000.04 & 11 & 1990 & 2046.52 & 19 & 1998 & 2506.00 & 27 & 2006 & 2720.00 \\
4 & 1983 & 1477.98 & 12 & 1991 & 1898.87 & 20 & 1999 & 2305.60 & 28 & 2007 & 2454.00 \\
5 & 1984 & 1634.46 & 13 & 1992 & 1840.30 & 21 & 2000 & 1638.00 & 29 & 2008 & 2840.00 \\
6 & 1985 & 1225.26 & 14 & 1993 & 1900.90 & 22 & 2001 & 1953.40 & 30 & 2009 & 2460.00 \\
7 & 1986 & 1397.71 & 15 & 1994 & 2015.70 & 23 & 2002 & 2214.80 & 31 & 2010 & 2842.50 \\
8 & 1987 & 1675.81 & 16 & 1995 & 1992.40 & 24 & 2003 & 2259.60 & 32 & 2011 & 3171.00 \\
\hline
\end{tabular}

\section{Results and Analysis}

Time Series Curve of Maize Yield. From the point of view of the distribution of the output of these years, we can not determine which kind of function model is more close to the sample data, so the application of a variety of curve estimation. Comprehensive comparative analysis and determination of the relatively more reasonable time series curve model. In this research, we choose one element linear, three order function, exponential function and power function to estimate the four kinds of curves. the estimated parameters of these four kinds of curves, the effect of the three curve estimation is optimal, so the choice of the three function Cubic to fit the observed values is more appropriate. Through the analysis and comparison of Table 1 above, the relationship between the total yield and time series of maize was obtained, and the conclusion of regression analysis was made by selecting the three function. Among them, $\mathrm{y}$ is representative of maize yield, $\mathrm{x}$ stands for time series year.After fitting the specific model is: $\mathrm{y}=476.708+62.157 \mathrm{x}-0.507 \mathrm{x} 2+0.010 \mathrm{x} 3$ 


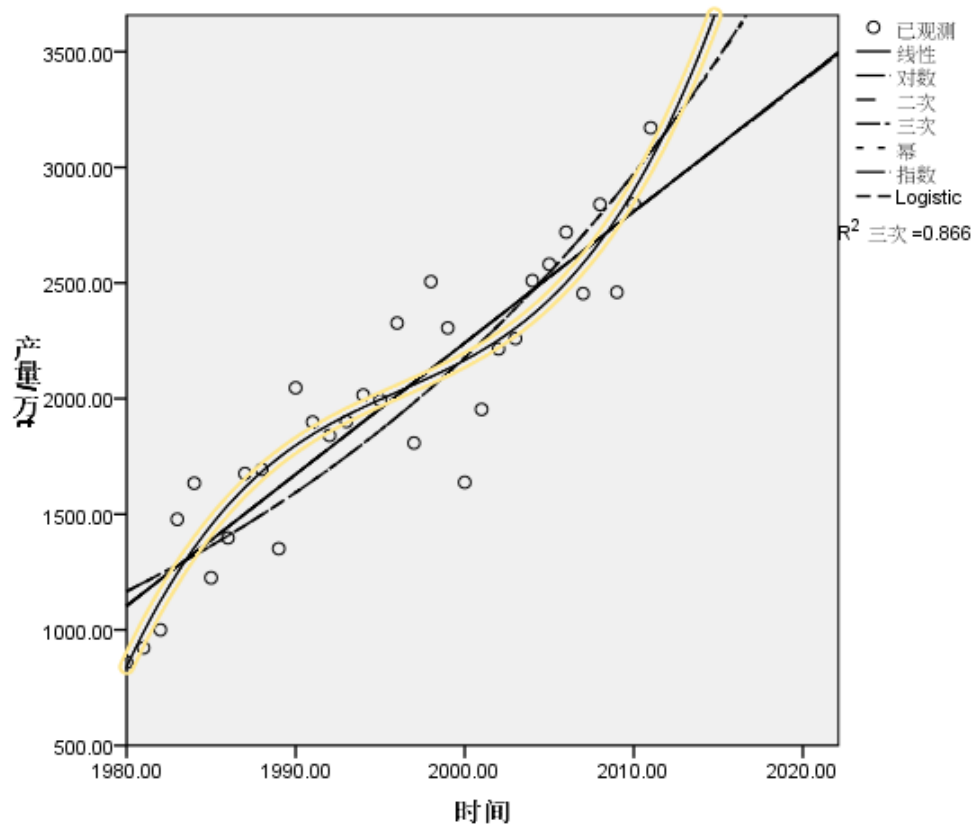

Figure 1. Contrast map of curve simulation and observation values

Dentification of ARIMA (P, D, q) Model of Maize Yield. From the data analysis, the time series distribution and the state of the output are shown in Fig. 2. In general, it is shown that there is an increasing trend of rising year by year. Therefore, when analyzing the output data, different methods are used for different requirements. Autocorrelogram to the original data of application software (ACF) and partial correlation graph (PACF), the original time series and the results display does not meet the stability requirements, necessary to deal with the data, here we use differential treatment process, so that the original time series stationary.

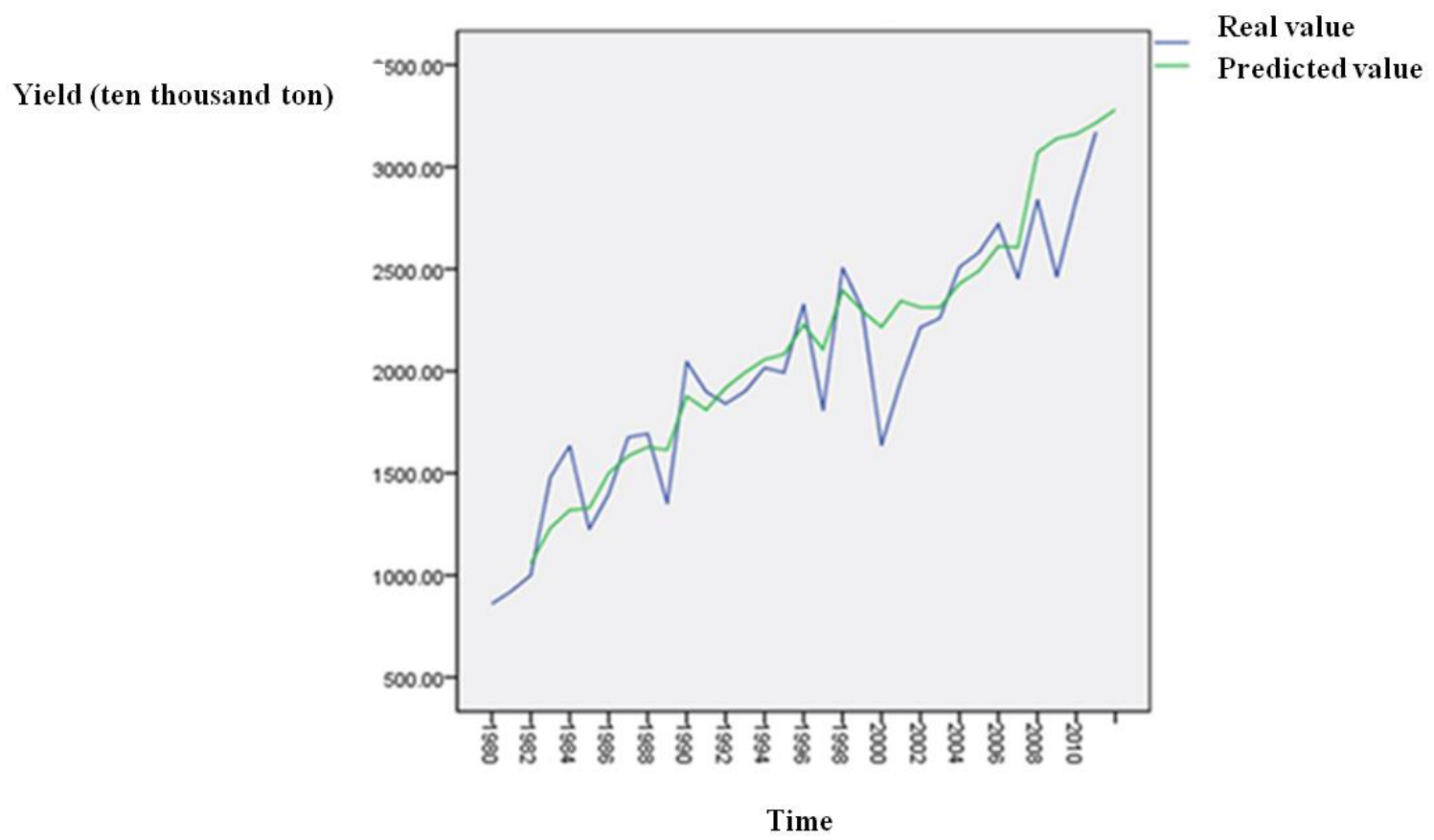

Figure 2. The yield forecast map

\section{Conclusions}

In the actual agricultural production process, the researchers know the time variability of soil nutrients, and on the other hand, it is also found that the crop yield in the study area also has 
obvious spatial and temporal variability. Zhang Xiaojie et al. Applied the traditional time series forecasting model and ARIMA model to predict the total grain yield in Shandong Province, and get the result that the regression equation is better than [8]. Of course, the variability of grain yield is affected by many factors, such as variety, environment, climate, pests, cultivation and management mode, through the analysis of the precise operation area in recent years, soil nutrient and yield data, change rule mining and grasp the crop yield in space and time. The temporal variation in crop yield model.

\section{Acknowledgements}

Funds for the research was provided by scientific research fund of Jilin Agricultural University (No.201620), Jilin province university undergraduate science and technology innovation fund. National university undergraduate science and technology innovation fund (No.201510193040).spark plan Ministry of science and technology (2015GA660004).

\section{References}

[1] Xu Junyan. Discussion on the application of regression analysis and forecasting method in the financial budget of the $[\mathrm{J}]$. financial sector, the 5 phase of the 2011 146-147

[2] C. Narendra Babu, B. Eswara Reddy .A moving-average filter based hybrid ARIMA-ANN model for forecasting time series data[J]. Original Research Article Applied Soft Computing, 2014, 23(10):27-38

[3] George C. Tiao Time Series: ARIMA Methods International Encyclopedia of the Social \& Behavioral Sciences (Second Edition), 2015, 316-321

[4] Wang Lijie, Liu Zhidong economic time series analysis technology in coal price forecasting [J]. Journal of China coal society, 2001, 26 (1): 109-112

[5] Zhu Dongmei, Huang Daichun, Hu Jianhua, Wang Qingxin, application of [J]. ARIMA time series model in budget revenues of telecom products in micro computer information, 2006, 22 (6-3): 178-179303

[6] Zhang Ya, Xiao Dongrong, Chen Keyan, Wang Dong. Prediction model of time series of Shenzhen's [J]. statistical analysis and decision, in 20085 33-34

[7] Xiao Xinru, Liu Xiaoxia. Study on regional population distribution and spatial correlation -Taking Anhui Province as an example [J]. Journal of Nanjing College for Population Program Management, 2013 4, 28-32,

[8] Ertur C,Koch W,Regional disparities in the European Union and the enlargement process:

[9] An exploratory spatial data analysis, 1995-2000. Annals of Regional science, 2006, 40(4): 723-765 